\title{
Reliability of symptoms to determine use of bone scans to identify bone metastases in lung cancer: prospective study
}

\author{
Martin Hetzel, Juergen Hetzel, Coskun Arslandemir, Karin Nüssle, Holger Schirrmeister
}

Based on the hypotheses that most skeletal metastases in lung cancer are clinically symptomatic, that the incidence of bony metastases in early stages is low, and that bone scintigraphy has a sensitivity of nearly $100 \%$, leading professional societies recommend diagnostic skeletal imaging depending on clinical symptoms. ${ }^{12}$ No study has assessed the significance of skeletal symptoms as a criterion for skeletal imaging in patients with lung cancer since $1991 .^{3}$ But in the intervening period gamma camera technology has been considerably refined and more sensitive methods such as magnetic resonance imaging have become available for skeletal imaging.

We redetermine the role of symptoms and serum concentrations in detecting bony metastases in lung cancer and reassess the accuracy of bone scans for screening.

\section{Participants, methods, and results}

From September 1999 to September 2001 we recruited 153 consecutive patients at University Hospital Ulm. We included patients based on cytological or histological evidence of lung cancer returned no more than 10 days before entry into the study. Of these, 121 (79\%; 88 men and 33 women; median age 66, range 40-83 years) agreed to participate. Exclusion criteria were a history of malignant disease, pregnancy, and age less than 18 years. All patients gave written informed consent. Diagnosis was non-small cell lung cancer in 84 patients and small cell lung cancer in 37 patients. We questioned and examined all patients about skeletal complaints. Physical examination included percussion, compression, flexion, extension, and rotation of the vertebral column and extremities and evaluations of patients' neurological status. We also measured serum calcium and alkaline phosphatase concentrations. New skeletal symptoms within the previous six months were judged as suspicious for bony metastases.

We did bone scans blinded to the history and findings of the physical examination. The combined results of magnetic resonance imaging of the vertebral column and patients' subsequent clinical course were the ideal for identification of bony metastases.

We found skeletal metastases in 40 patients (33\%). Incidence was nearly identical at 33\% (28) in patients with non-small cell lung cancer and 32\% (12) in those with small cell lung cancer. These patients had normal serum alkaline phosphatase and calcium concentrations. Three quarters (91) of patients had symptoms. In only $19 \%$ (23) of patients with symptoms did the location of metastases correspond to the symptoms. Routine bone scans correctly identified skeletal metastases in 29 patients (sensitivity 73\%; 95\% confidence interval $56 \%$ to $85 \%$ ). Bone scans were correctly negative in 80 of 81 patients (specificity 99\%; 93\% to 100\%). If bone scans were done in only the 91 patients reporting skeletal complaints, the sensitivity would have been

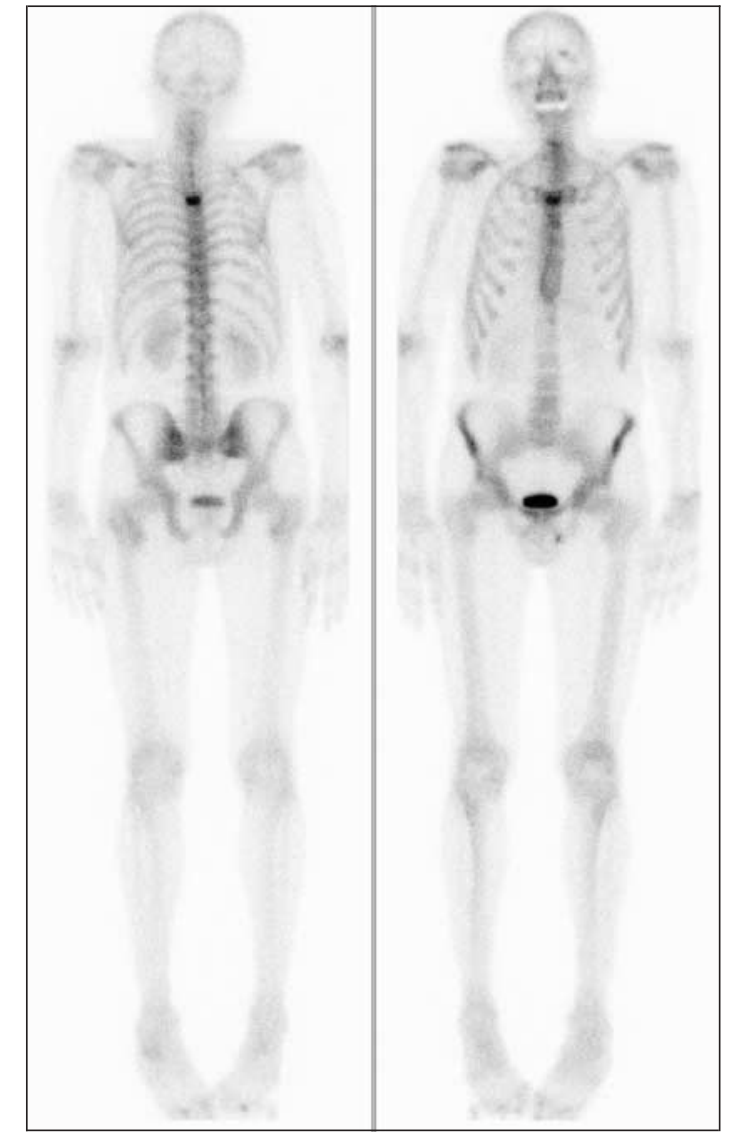

Department of Internal Medicine II, University of Ulm, D-89081 Ulm, Germany

Martin Hetzel consultant physician Juergen Hetzel specialist in internal medicine

Department of Nuclear Medicine University of Ulm

Coskun Arslandemir

physician

Holger

Schirrmeister consultant in nuclear medicine

Department of Diagnostic

Radiology,

University of Ulm

Karin Nüssle

radiologist

Correspondence to: M Hetzel

martin.hetzel@

medizin.uni-ulm.de

BMJ 2004;328:1051-2

Bone scan showing a 49 year old white male in good general condition (Eastern Cooperative Oncology Group performance status 0 ) diagnosed with non-small cell lung cancer (cT3N2M0). He reported pain in the shoulder joints of two years' duration. Physical examination failed to show pain on percussion, pressure, or movement of the vertebral column. His complaints were correctly interpreted by the examiner as being arthritic in origin. A bone scan got despite the examiner's interpretation of the patient's complaints identified an asymptomatic metastasis localised to the fourth thoracic vertebra. The correct interpretation of the patient's symptoms and the resulting decision to defer skeletal scintigraphy would have represented false negative findings and would have resulted in the patient undergoing neoadjuvant treatment

reduced to 53\%. A further restriction of the method to those 23 patients with suspicious complaints would have resulted in a further reduction in sensitivity to $20 \%$ (8 patients).

\section{Comment}

Only a small proportion of bone metastases can be recognised based on clinical symptoms or by increased serum alkaline phosphatase or calcium levels. Because of the high incidence of arthritic complaints, ${ }^{4}$ guidelines mandating bone scans only in patients with skeletal complaints often result in coincidental discovery of bony metastases (figure). Restricting bone scans to patients whose skeletal complaints were clinically 
suspicious for metastatic disease, however, would have resulted in an unacceptably low sensitivity of $20 \%$. Considering the high incidence of bone metastases at initial diagnosis, this might lead to a dramatic increase in the number of patients undergoing futile surgery or neoadjuvant chemotherapy.

Contributors: MH had the original idea, designed the study, selected the patients, managed the study, interpreted the results, wrote the first draft of the paper, and edited the paper. JH gathered, interviewed, and examined the patients. CA interpreted the bone scans; gathered, interviewed, and physically examined the patients; interpreted the results, and prepared the manuscript. $\mathrm{KN}$ interpreted the magnetic resonance images. HS had the original idea, interpreted the bone scans, and prepared the manuscripts. MH and HS are guarantors.
Funding: None.

Competing interests: None declared.

Ethical approval: University of Ulm.

1 American Thoracic Society, European Respiratory Society. Pretreatment evaluation of non-small-cell lung cancer. Am J Respir Crit Care Med 1997;156:320-32.

2 Silvestri GA, Tanoue LT, Margolis ML, Barker J, Detterbeck F. The noninvasive staging of non-small cell lung cancer: the guidelines. Chest 2003; $123:$ S147-56.

3 Michel F, Soler M, Imhof E, Perruchoud AP. Initial staging of non-small cell lung cancer: value of routine radioisotope bone scanning. Thorax 1991;46:469-73.

4 Deyo RA, Tsui-Wu Y-J. Descriptive epidemiology of low-back pain and its related medical care in the United States. Spine 1987;12:264-8.

(Accepted 11 November 2003)

\section{Trends in prevalence of symptoms of asthma, hay fever, and eczema in 12-14 year olds in the British Isles, 1995-2002: questionnaire survey}

H Ross Anderson, Ruth Ruggles, David P Strachan, Jane B Austin, Michael Burr, David Jeffs, Peter Standring, Andrea Steriu, Rosie Goulding

Department of

Community Health Sciences, St

George's Hospital

Medical School,

London SW17 0RE

H Ross Anderson

professor of

epidemiology and

public health

Ruth Ruggles

specialist registrar in

public health medicine

David P Strachan

professor of

epidemiology

Highlands and

Islands Health

Research Institute,

University of

Aberdeen,

Inverness IV2 3ED

Jane B Austin

paediatrician and

honorary lecturer

Department of

Epidemiology,

Statistics and Public

Health, University

of Wales College of

Medicine Cardiff

CF14 4XN

Michael Burr

reader in

epidemiology

Board of Health,

St Martins,

Guernsey

GY4 6UU

David Jeffs

director of public

health

Princess Elizabeth Hospital, St Martins

Peter Standring

paediatrician

continued over

BMJ 2004;328:1052-3
The prevalence of symptoms of asthma, allergic rhinitis, and atopic eczema in children in the United Kingdom ranks among the highest in the world. ${ }^{1}$ The evidence from most repeat surveys is that prevalence has increased over the past three decades, ${ }^{2}$ but the most recent of these studies observed that from 1991 to 1998 the increase was confined to milder symptoms of asthma. ${ }^{3}$ Since the early $1990 \mathrm{~s}$ the incidence of asthma episodes presenting to general practitioners, and of hospital admissions, has fallen substantially. ${ }^{2}$

\section{Participants, methods, and results}

In 1995, as part of the international study of asthma and allergies in childhood (ISAAC), we surveyed symptoms of atopic disease in England, Scotland, Wales, and the offshore islands of Guernsey, Isle of Man, and Jersey. ${ }^{45}$ A self completed questionnaire which adhered to the core ISAAC protocol was administered to secondary school children aged 12-14 in school years 8 and 9 (S2 and S3 in Scotland). In 2002 the survey was repeated in Scotland, Wales, and the islands in the same school years, using the same questionnaire and procedures in the same period of the year and, mostly, in the same schools. In England, only the schools in the South East Region were surveyed a second time. The table shows the changes in prevalence over the seven years from 1995 to 2002 .

Overall, the prevalence of any wheezing or whistling in the chest in the past 12 months fell from $34 \%$ to $28 \%$ (19\% relative reduction). Even larger proportional falls were observed for frequent attacks $(35 \%)$ and speech limiting attacks (24\%). Large reductions were also observed for symptoms of allergic rhinoconjunctivitis $(16 \%)$ and atopic eczema (30\%). The proportion of children reporting "ever" having had "asthma" or "eczema" increased (26\% and 15\%), as did the lifetime prevalence of "hay fever" $(8 \%)$. Trends in the four regions were similar.

The fall in prevalence is consistent with other sources. From 1995 to 2000, hospital admissions for asthma fell by $20.4 \%$ in 10-14 year olds in England, Scotland, and Wales combined (see table). From 1995 to 2002, in 10-14 year olds in a sample of 75 English and Welsh practices, visits to a general practitioner for episodes of asthma decreased by $47 \%$ (from 38.3 to 20.4 per 1000). Recently released data from the health survey for England shows that from 1997 to 2001, the 12 month prevalence of wheezing in 10-14 year olds, based on parental reporting, fell by $18 \%$ (from $17.4 \%$ to $14.2 \%)$.

\section{Comment}

The burden of self reported asthma and other allergic diseases among adolescents has changed substantially for the better in recent years throughout the British Isles. These trends correspond to those seen in the 10-14 year age group in hospital admissions, consultations with general practitioners, and parentally reported symptoms in the health survey for England. Just as we do not know why the prevalence of symptoms of asthma has increased since the 1950 s, we do not know why it should now be decreasing. The increased use of effective treatment, especially inhaled steroids, is likely to have been important in reducing the severity of episodes, but this is unlikely to explain the decrease in mild wheeze symptoms, which is more consistent with a fall in the underlying prevalence. The

This article was posted on bmi.com on 17 March 2004: http://bmj.com/ cgi/doi/10.1136/bmj.38057.583727.47 